\title{
COMPACT MODEL SYNTHESIS FOR PARTIALLY OBSERVED OPERATIONAL SYSTEMS
}

\author{
Jean-Luc DION \\ SUPMECA - EA 2336 \\ Paris - France \\ Nicolas PEYRET \\ SUPMECA - EA 2336 \\ Paris - France
}

\author{
Fatma ABID \\ SUPMECA - EA 2336 \\ Paris - France \\ Franck RENAUD \\ SUPMECA - EA 2336 \\ Paris - France
}

\author{
Gaël CHEVALLIER \\ SUPMECA - EA 2336 \\ Paris - France
Moustafa SEIFEDDINE
SUPMECA - EA 2336 \\ Paris - France
}

\author{
Hugo FESTJENS \\ SUPMECA - EA 2336 \\ Paris - France
}

\author{
Cyrille STEPHAN \\ ONERA \\ Paris - France
}

\begin{abstract}
This work proposes a Compact Model Synthesis (CMS) for Partially Observed Operational Systems (POOS) without using the complete knowledge of models. Series of "grey boxes" fed with partial observations are built in order to synthesize target variables with compact models. The recursive process for real time computation is based on Kalman Filters (KF). This stochastic approach allows to converge in line toward deterministic models with estimated uncertainties and without intrusion on the complete model process. Mathematical context is described first and illustrated secondly with two examples.
\end{abstract}

\section{ACRONYMS}

Compact Model Synthesis (CMS)

Continuous State Systems (CSS)

Discrete Linear State Systems (DLSS)

Discrete Non Linear State Systems (DNLSS)

Extended Kalman Filters (EKF)

Finit Element Simulation (FES)

Kalman Filters (KF)

Linear Time-Invariant (LTI)

Ordinary Differential Equations (ODE)

Operating Modal Analysis (OMA)

Parametric Grey Boxes (PGB)

Partially Observed Operational Systems (POOS)

Reduced Order Models (ROM)

Series of Elementary Generic Models (SEGM)

Unscented Kalman Filters (UKF)

\section{INTRODUCTION}

Reduced Order Models (ROM) are generally built in order to make simulation processes faster.
These methods are very useful as part of an optimization process using a High Fidelity Finite Element Model parameterized by design variables or as part of the acceleration process of simulations with a high number of Degrees Of Freedom. Both objectives are quite different insofar as, for optimization, it is interesting to build parametric models of small size; while for acceleration, design parameters are useless but accuracy is the main goal. We focus on the framework of optimization even if this paper refers only to the modeling step.

Numerous methods are available to achieve this goal. We can classify the methods of Model Reduction into two families: the first ones aims to provide a basis, spanning a subspace which belongs to the space of solutions, to link the variables of the Full Order Models (FOM) to a smaller number of variables; Such methods are often called Ritz methods or "kinematic methods" (KM); The second methods aims to identify a meta-model able to provide accurate results for selected degrees of freedom of the FOM; In the following, we will call this methods "Identification Methods" (IM). Sometimes, in an optimization framework, both kinds of methods are used: the first one to reduce the number of variables, the second one to build a parametric reduced order model, see for example R. Filomeno et al. [1]. To reduce the complex systems constituted with several components, it is rather commonplace to reduce each component with $\mathrm{KM}$ and to make the synthesis of the system, see for example Craig [2] or Guyan [2]. The Way to Build Ritz base has been widely discussed in the previous work and we can consider two methods: modal approaches and optimal approaches based on proper orthogonal decomposition, see for example Balmès [4]. The non-linear problems involve specific Model Order Reduction Techniques, see 
for example Kerschen et al. [5]. The final challenge is to build ROM parameterized by design variables, see for instance Amsallem et al. [6] or Balmès [7].

This work purpose is to build ROM based on observation of operating systems. "Operating Systems" could be models during simulations such as thermal Finite Element Models (FEM) [8] or operating experimental devices such as jointed structures in free vibration [9] and [10] or motion picture of mechanical system. Proposed ROM are based on Series of Elementary Generic Models (SEGM) considered as Parametric Grey Boxes (PGB) which had to be identified during operating process. A priori, the length of SEGM is unknown but the nature of PGBs is chosen regarding the observed phenomenon. For example, thermic study are described with first order Ordinary Differential Equations (ODE) in PGBs whereas dynamics are described with second order ODE. Operating conditions are often imposed for several problems: Operating Modal Analysis (OMA) of helicopters or Energy Production Power Supply is performed during operating conditions. Survey and diagnostic for help to decision in automated manufacturing are sometimes carried out in real time in the process. Motion picture of mechanical scenario or FEM are composed of large number of observable variables. In that case, ROMs built in real time allow saving computational time and allocated memory. For these reasons KF are retained in the recursive parametric identification process of PGBs.

\section{KALMAN FILTERS AND SERIES OF ELEMENTARY GENERIC MODELS}

CMS is performed with the help of three different kinds of $\mathrm{KF}$ [11] regarding the nature of analytical models in PGMs. "Classical" Kalman Filters are convenient for Discrete Linear State Systems (DLSS), Extended Kalman Filters (EKF) for Discrete Non Linear State Systems (DNLSS) and Unscented Kalman Filters (UKF) for systems described with Continuous State Systems (CSS) as complex dynamic non linear systems, non autonomous systems or time dependant holonomic systems.

Three kinds of bases are proposed For DLSS (KF) and DNLSS (EKF) : Generalized Fourier Series (GFS) with harmonic and non harmonic spectral components (used for second order systems and for moving trackers on moving targets), exponential bases for first order systems such as relaxation phenomenon, plasticity or thermodynamics and polynomial bases for ballistics or unexpected non autonomous systems. The two first kind of series (sinusoids bases and exponential bases) are detailed later in this paper.

\subsection{State Systems}

Three kind of state systems are studied in this paper. The first one concerns DLSS which could be writen as:

$X_{n+1}=F_{n} \cdot X_{n}+B_{n} \cdot U_{n}+W_{n}$

$Z_{n}=H_{n} \cdot X_{n}+V_{n}$

where :

$X$ State variables

$F$ State matrix model

$B$ Control matrix model

$U$ Control variable vector

$W$ Process noise

$Z$ Observation vector

$H$ Observation matrix

$V$ Observation noise

$n$ time increment

When the discrete system could not be written as a linear system (with invariant matrix) the DNLSS is assumed as :

$$
\begin{aligned}
& X_{n+1}=\Phi\left(X_{n}, U_{n}\right)+W_{n} \\
& Z_{n}=H\left(X_{n}\right)+V_{n} \\
& \text { with : } \\
& \phi \quad \text { Non linear state function } \\
& H \quad \text { Non linear observation function }
\end{aligned}
$$

In the case of linear continuous time systems the equations are :

$$
\begin{aligned}
& \dot{X}(t)=F \cdot X(t)+B \cdot U(t)+w(t) \\
& Z(t)=H \cdot X(t)+D \cdot U(t)+v(t)
\end{aligned}
$$

For non linear systems, CSS is described in a general sense as follows :

$$
\begin{aligned}
& \dot{X}(t)=\Phi(X(t), U(t))+w(t) \\
& Z(t)=H(X(t), U(t))+v(t)
\end{aligned}
$$

All these systems are suitable for KF, EKF or UKF.

\subsection{Kalman Filters}

The KF and EKF has been widely used over the past decades in the case of discrete systems. In this formulation the state variables are extended to the parameters of the PGBs that are to be identified. It allows to compute an estimation of the PGBs in the case where the shape of the solution of the problem is already kwown. For instance, in the case of oscillating systems, the time evolution is pseudo-harmonic (see section 2.3); in the case of relaxation system, such as thermodynamics systems, the evolution is pseudo-exponential (see section 2.4). The incrementation of the EKF requires the linearization of the estimated evolution which can lead to a strong approximation in the case of non-smooth non-linear systems. In order to avoid this linearization process Julier and Uhlmann (1997) have developed the "unscented kalman filter" UKF [13]. In that method, the estimation of 
the state variables and their variances are directly incremented thanks to the unscented transformation (UT). This function can be more generally used to estimate the result of applying a given nonlinear transformation to a probability distribution. The two first moments of the distribution are simply propagated by applying the nonlinear function to a set of point referred to as sigma points. Eliminating the need for linearization also provides advantages independent of any improvement in estimation quality. One immediate advantage is that the UT can be applied to identify the parameters of a Non-linear ODE for which the solution cannot be analytically solved. Practically, the implementation of the UKF is also easier than EKF.

2.3. Series of Elementary Generic Models and bases : oscillating systems

Second order ODEs often leads to oscillating solutions. In the case of non linear systems oscillating solutions are amplitude and frequency modulated.

A sinusoid whose amplitude and frequency are modulated over time can be described in the complex domain as an analytic signal :

$$
x(t)=a(t) \exp (j \phi(t))
$$

where $\mathrm{a}(\mathrm{t})$ is the instantaneous complex amplitude and $\Phi(t)$ is the instantaneous phase. The discrete form of $x(t)$ at the time step $t_{n}=n \Delta t$ is $x_{n}=x(n \Delta t)$. The complex variable $x_{n}$ can be divided into its real and imaginary parts $x_{n}=x_{1, n}+j x_{2, n}$. A sinusoid that slightly varies over time can be approximated by :

$$
x_{n}=a_{n} \exp \left(j\left(2 \pi f_{n} n \Delta t\right)\right)
$$

where $f_{n}$ is the instantaneous frequency.

As the parameters fn and an of the sinusoid slightly vary over time, they are almost equal between two consecutive time steps. Then a transition formulation can be given from $x_{n}$ to $x_{n+1}$ :

$$
x_{n+1} \approx a_{n} \exp \left(j\left(2 \pi f_{n} n \Delta t\right)\right) \times \exp \left(j\left(2 \pi f_{n} \Delta t\right)\right)
$$

This approximation is only true if modulations of an and fn are slower that the period of the sinusoid. This constraint is assumed to be verified. Then a linear transition is obtained between the imaginary part $x_{2}$,. and real part $x_{1}$. of $x_{n}$ and $x_{n+1}$ :

$$
\left(\begin{array}{l}
x_{1, n+1} \\
x_{2, n+1}
\end{array}\right)=\left[\begin{array}{cc}
\cos \left(2 \pi f_{n} \Delta t\right) & -\sin \left(2 \pi f_{n} \Delta t\right) \\
\sin \left(2 \pi f_{n} \Delta t\right) & \cos \left(2 \pi f_{n} \Delta t\right)
\end{array}\right]\left(\begin{array}{l}
x_{1, n} \\
x_{2, n}
\end{array}\right)(13)
$$

This formulation choice allows keeping the matrix Fn linear and time-independent if the signal is only amplitude modulated and not frequency modulated (the frequency fn keeps constant).

The instantaneous amplitude an is .

$$
a_{n}=\sqrt{x_{1, n}^{2}+x_{2, n}^{2}}
$$

As an and fn should be allowed to vary over time, it is proposed here to use the following non linear state space formulation :

$X_{n+1}=\Phi\left(X_{n}\right)+W_{n}$

where $X_{n}=\left(\begin{array}{lll}x_{1, n} & X_{2, n} & X_{3, n}\end{array}\right)^{T}$ and $W_{n}$, the process noise. A state variable $x_{3, n}=2 \pi f_{n} \Delta t$ was added to track the evolution of the instantaneous frequency fn. The transition between two time steps is composed by a sum of two parts: the stationary part and the evolutionary part.

The stationary part links two successive points of a stationary sinusoid by $\Phi($.$) . Then \Phi($.$) is assumed as the$ non-linear transition function and is given by $\Phi\left(X_{n}\right)=F_{n} X_{n}$

where $\quad F_{n}=\left(\begin{array}{ccc}\cos \left(x_{3, n}\right) & -\sin \left(x_{3, n}\right) & 0 \\ \sin \left(x_{3, n}\right) & \cos \left(x_{3, n}\right) & 0 \\ 0 & 0 & 1\end{array}\right)$

The two first components are related to the complex amplitude and are obtained by the previous linear relation. The third component $\mathrm{x}_{3, \mathrm{n}+1}=\mathrm{x}_{3, \mathrm{n}}$ constrains the frequency not to change strongly between two time steps.

Up to now, the non-stationary behavior of the sinusoid was not modeled because it is not possible to express an exact equation for this evolution. We suppose that $\mathrm{W}_{\mathrm{n}}$ is a random variable whose probability law is Gaussian : $\mathrm{W}_{\mathrm{n}}=\mathrm{N}(0, \mathrm{Q})$, where $\mathrm{Q}$ is its variance matrix. Then the variations of amplitude and frequency are allowed by random values of $\mathrm{W}_{\mathrm{n}}$.

In a first glance, it could seem strange to choose a random variable for an effect which is generally deterministic. For instance, the variation of frequency excitation of an engine is mainly deterministic. Anyway, this state space does not need to represent accurately the evolution of a sinusoid on a long period, but only step by step. Then on a short time scale, a random evolution of an and fn is enough to model a non-stationary sinusoid.

For a signal composed of $\mathrm{M}$ observable modulated sinusoidal components, the size of the ROM is $M$ and the size of the state function is $3 \mathrm{M}$.

and

$$
F_{n}\left(X_{n}\right)=\left[\begin{array}{ccccccc}
\cos \left(x_{3, n}\right) & -\sin \left(x_{3, n}\right) & 0 & \ldots & 0 & 0 & 0 \\
\sin \left(x_{3, n}\right) & \cos \left(x_{3, n}\right) & 0 & \ldots & 0 & 0 & 0 \\
0 & 0 & 1 & & 0 & 0 & 0 \\
\vdots & \vdots & & \ddots & 0 & 0 & 0 \\
0 & 0 & 0 & \ldots & \cos \left(x_{3+3(M-1, n)}\right) & -\sin \left(x_{3+3(M-1, n)}\right) & 0 \\
0 & 0 & 0 & \ldots & \sin \left(x_{3+3(M-1), n}\right) & \cos \left(x_{3+3(M-1), n}\right) & 0 \\
0 & 0 & 0 & \ldots & 0 & 0 & 1
\end{array}\right]
$$

and

$$
X_{n}=\left(\begin{array}{c}
x_{1, n} \\
x_{2, n} \\
x_{3, n} \\
\vdots \\
x_{1+3(M-1), n} \\
x_{2+3(M-1), n} \\
x_{3+3(M-1), n}
\end{array}\right)
$$


This state space formulation is non linear: the state matrix $F_{n}\left(X_{n}\right)$ is varying over time and depends on the frequency modulation.

In reality, only the real part $x_{1, n}$ of the analytic signal $x_{n}$ can be observed. Unlike the transition phase, the observation phase is completely linear

$$
\begin{aligned}
& Z_{n}=\left[\begin{array}{ccc}
1 & 0 & 0
\end{array}\right] X_{n}+V_{n} \\
& Z_{n}= \\
& X_{1, n}+V_{n}
\end{aligned}
$$

for one sinusoid and slightly more complex for $M$ sinusoids

$$
\begin{aligned}
& Z_{n}=\left[\begin{array}{lllllll}
1 & 0 & 0 & \ldots & 1 & 0 & 0
\end{array}\right] X_{n}+V_{n} \\
& Z_{n}=
\end{aligned}
$$

where $V_{n}$ is a noise observation random process.

Finally, a nonlinear discrete state space model has been derived to model the transition and observation of sinusoid components mixed with random processes

$$
\left\{\begin{array}{l}
X_{n+1}=\Phi\left(X_{n}\right)+W_{n} \\
Z_{n+1}=H\left(X_{n+1}\right)+V_{n+1}
\end{array}\right.
$$

where $\Phi($.$) is the nonlinear transition function given by$ $\Phi\left(X_{n}\right)=F\left(X_{n}\right) X_{n}$ and $H($.$) is the linear observation function$ given by :

$H\left(X_{n+1}\right)=\left[\begin{array}{llllllll}1 & 0 & 0 & \ldots & 1 & 0 & 0\end{array}\right] X_{n+1}$.

2.4. Series of Elementary Generic Models and bases : non oscillating systems

When the system is compose with first order ODEs or assumed as a sum of monotone responses, series of negative exponential are used. The continous state equation of the elementary generic model with control is

$$
x(t)=\left(x_{0}-a U\right) \exp \left(-\delta\left(t-t_{0}\right)\right)+a U
$$

With :

$x_{0}, t_{0}$ assumed as initial conditions, $a$ and $\delta$ are constant to be identified.

The DLSS of this equations is written as :

$$
x_{n+1}=x_{n} \mathrm{e}^{-\delta \Delta t}+U_{n} a\left(1-\mathrm{e}^{-\delta \Delta t}\right)
$$

With $\Delta \mathrm{t}$ the time increment.

The DNLSS of this equations is written as :

$$
x_{n+1}=x_{n} \mathrm{e}^{-\delta_{n} \Delta t}+U_{n} a_{n}\left(1-\mathrm{e}^{-\delta_{n} \Delta t}\right)
$$

The complete SEGM is built as follows for the non linear state matrix and control matrix :

$$
F_{n}\left(X_{n}\right)=\left[\begin{array}{ccccccc}
\mathrm{e}^{-x_{2, n} \Delta t} & 0 & 0 & \ldots & 0 & 0 & 0 \\
0 & 1 & 0 & \ldots & 0 & 0 & 0 \\
0 & 0 & 1 & & 0 & 0 & 0 \\
\vdots & \vdots & & \ddots & 0 & 0 & 0 \\
0 & 0 & 0 & \ldots & \mathrm{e}^{-x_{2+3(M-1), n} \Delta t} & 0 & 0 \\
0 & 0 & 0 & \ldots & 0 & 1 & 0 \\
0 & 0 & 0 & \ldots & 0 & 0 & 1
\end{array}\right]
$$

and

$$
B_{n}\left(X_{n}\right)=\left[\begin{array}{ccccccc}
x_{3, n}\left(1-\mathrm{e}^{-x_{2, n} \Delta t}\right) & 0 & 0 & \ldots & 0 & 0 & 0 \\
0 & 0 & 0 & \ldots & 0 & 0 & 0 \\
0 & 0 & 0 & & 0 & 0 & 0 \\
\vdots & \vdots & & \ddots & 0 & 0 & 0 \\
0 & 0 & 0 & \ldots & x_{3, n}\left(1-e^{-x_{2}+3(M-1), n}, a^{t}\right) & 0 & 0 \\
0 & 0 & 0 & \ldots & 0 & 0 & 0 \\
0 & 0 & 0 & \ldots & 0 & 0 & 0
\end{array}\right]
$$

The observation equation is the same for oscillating an non oscillating systems. and the complete DNLSS is :

$$
\left\{\begin{array}{llc}
X_{n+1} & = & F_{n}\left(X_{n}\right)+B_{n}\left(X_{n}\right) U_{n}+W_{n} \\
Z_{n+1} & = & H\left(X_{n+1}\right)+V_{n+1}
\end{array}\right.
$$

\section{APPLICATIONS}

Two kinds of applications are presented. The first one concerns oscillating systems with amplitude and frequency modulated signals tracked with EKF for CMS.

The second study is in the field of thermodynamic and is treated with UKF.

3.1. Oscillating systems and solutions of second order ODEs

Tracked signals are amplitude and frequency modulated. The CMS is performed with SEKF based on SEGM. The expected ROM is composed with two EGM. The first observed component is around $22 \mathrm{~Hz}$ and the second around $50 \mathrm{~Hz}$. For each component, the amplitude modulation is determined with the instantaneous amplitude:

$$
a_{n}=\sqrt{x_{1, n}^{2}+x_{2, n}^{2}}
$$

This amplitude could also be obtained by the complex analytic signal composed of the observed signal (real part) and its Hilbert transform (imaginary part).

The frequency modulation is determined by the instantaneous frequency :

$$
f_{n}=\frac{x_{3, n}}{2 \pi \Delta t}
$$

For each tracked signal, spectral amplitudes show the main component and lateral modulated bands (Figure 1). 


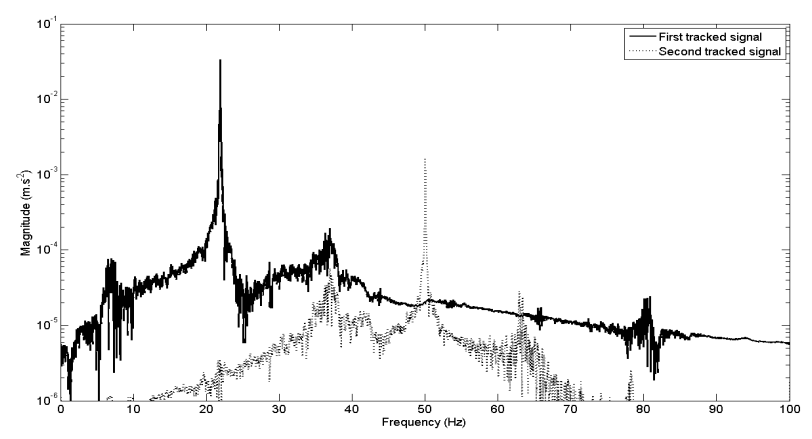

Figure 1 : Spectrums of two tracked signals.

Symmetric lateral bands are due to modulation effects on spectrums

The first tracked signal is frequency and amplitudemodulated (figure 1 and figure 2). The second tracked signal is only amplitude-modulated and not frequencymodulated.

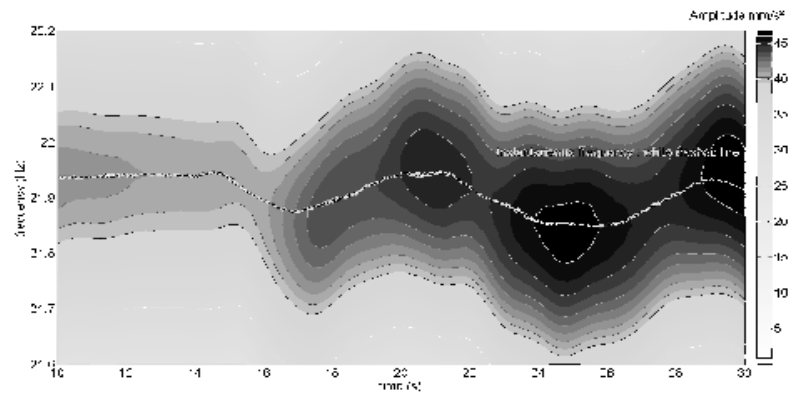

Figure 2 : Time-frequency representation of the first tracked signal. Magnitude and frequency modulation are identified with a good accuracy. (Instantaneous frequency is identified with 4 significant digits).

\subsection{Continuous State Systems}

System identification has been based largely on discretetime models for a long time in the past, ignoring certain merits of the native continuous-time models.

In this section, we are interested in the procedure of determining directly a continuous-time model of a dynamical system from observed data. That means, the differential-equation model is valid whatever the command nature or sampling data considered. We will consider first linear time-invariant (LTI) continuous system. As a simple illustration of this stage, let us consider a first-order system described by a CLSS as follow:

$$
\begin{aligned}
& \{\dot{x}(t)\}=[F]\{x(t)\}+[B] u(t) \\
& \{z(t)\}=[C]\{x(t)\}
\end{aligned}
$$

In particular:

$$
\begin{aligned}
& \{\dot{x}(t)\}=\left\{\begin{array}{l}
\dot{x}_{1}(t) \\
\dot{x}_{2}(t)
\end{array}\right\}=\left[\begin{array}{ll}
a_{1} & \\
& a_{2}
\end{array}\right]\{x(t)\}+\left[\begin{array}{l}
b_{1} \\
b_{2}
\end{array}\right] u(t) \\
& \{z(t)\}=\left\{\begin{array}{l}
z_{1}(t) \\
z_{2}(t)
\end{array}\right\}=\left[\begin{array}{ll}
c_{11} & c_{12} \\
c_{21} & c_{22}
\end{array}\right]\{x(t)\}
\end{aligned}
$$

where $\mathrm{X}(\mathrm{t})$ is the state vector, $\mathrm{Y}(\mathrm{t})$ the observation vector, $\mathrm{U}(\mathrm{t})$ the input vector, a1 and a2 invariant parameter system, b1 and b2 the parameters that locate the input vector to the state vector variables, and c11, c12, c21 and c22 the observation parameters.

The conversion of the Continuous system (30) into a recursive discrete system is performed using either EKF by means of exponential discretization technique [12], (Annex A) or UKF using an implicit numerical integration method named Dormand-Prince method [13], [14].

The resulting discrete-time Kalman model takes the following form:

$$
\begin{aligned}
& X_{k}=\left\{\begin{array}{l}
x_{k} \\
\theta_{k}
\end{array}\right\}=\left[\begin{array}{l}
\phi_{k}\left(x_{k-1}, u_{k-1}, \theta_{k-1}\right) \\
\theta_{k-1}
\end{array}\right] \\
& Z^{k}=\left\{\begin{array}{l}
z_{1, k} \\
z_{2, k}
\end{array}\right\}=H_{k}\left(x_{k}, \theta_{k-1}\right)
\end{aligned}
$$

Where the $\phi^{k}$ and $H^{k}$ are nonlinear evolution and observation functions at time $\mathrm{k}$, the $\theta^{k}$ a stationary parameter vector at time $\mathrm{k}$.

$$
\begin{aligned}
& X_{E K K_{k}}={ }^{t}\left[x_{1, k} x_{2, k}\left(e^{a 1 \Delta T}\right)_{k}\left(e^{a 2 \Delta T}\right)_{k}\left(\frac{b_{1}}{a_{1}}\right)_{k}\left(\frac{b_{2}}{a_{2}}\right)_{k} c_{11, k} c_{12, k} c_{21, k} c_{22, k}\right] \\
& X_{U K F, k}={ }^{t}\left[x_{1, k} x_{2, k} a_{1, k} a_{2, k} b_{1, k} b_{2, k} c_{11, k} c_{12, k} c_{21, k} c_{22, k}\right]
\end{aligned}
$$

\section{Numerical results}

This section presents numerical simulation results aiming to evaluate the performance of the EKF and UKF methods regarding system identification based on data produced numerically by the reference model (30). The forcing term, $\mathrm{u}$, is a square signal.

In the reference problem, (30) and (31), the terms $[\mathrm{F}],[\mathrm{B}]$ and $[\mathrm{C}]$ are assumed known and $\{\mathrm{x}\}$ is determined through a 
numerical integration method implemented in Matlab (Ode23; Runge-Kutta method).

In the Figure 3 and 4, we compare the response of the reference model (31) at points observation, $\mathrm{Z}$, to the response of the identified system model (33), $Z_{k}$, using Kalman filters, EKF and UKF, respectively, to evaluate its accuracy at five different times, $t \approx 2 s, 4 s, 6 s, 8 s, 10 s$.

Both EKF and UKF produce a good accuracy with almost equivalent convergence speed in that case, e.g. CLSS.

In the case of continuous non linear system, such as timedepend parameters $a_{1}$ and $a_{2}$, only UKF is appropriate. To process it, we use the same model (30) and the UKF algorithm is unchanged in comparison with linear case. Here, the advantage of UKF as regards the implementation simplicity is highlighted with respect to the EKF. This latter methodology actually is not extended to the processing of nonlinear continuous systems due to the difficulty related to the analytical discrete model construction step starting for CNLSS.
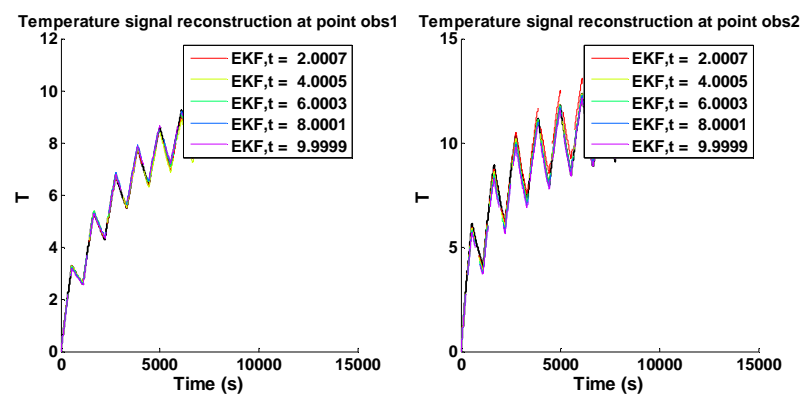

Figure 3: Comparison of response of reference model (black solid curve) to the response of identified system model at five times (starting points) using EKF; left, point observation 1, and right, point observation 2.
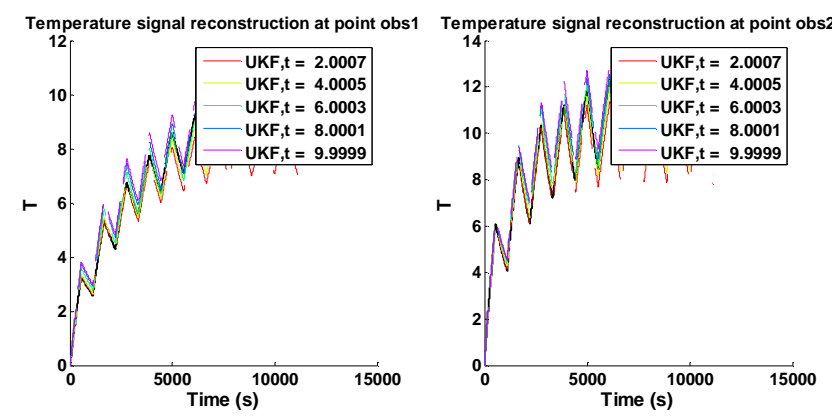

Figure 4: Comparison of response of reference model (black solid curve) to the response of identified system model at five times using UKF; left, point observation 1, and right, point observation 2.

\section{CONCLUSION}

Kalman Filters have been used successfully for compact model synthesis of oscillating and non oscillating systems.
The proposed method could be performed in line and with only few information about the complete model. The main challenge today is to work out an automatic method for initializing $\mathrm{KF}$ with robustness.

\section{REFERENCES}

[1] Filomeno Coelho R., Breitkopf P., Knopf-Lenoir, C. Model reduction for multidisciplinary optimization - Application to a 2D wing, Structural and Multidisciplinary Optimization, 10.1007/s00158-007-0212-5, 2007.

[2] Craig, R. R. Jr., "A Review of Time-Domain and Frequency Domain Component Mode Synthesis Methods," Int. J. Anal. and Exp. Modal Analysis, 1987, 2-2, pp 59-72

[3] Guyan RJ. Reduction of stiffness and mass matrices. AIAA Journal 1965; 3(2):380.

[4] Balmès, E., "Optimal Ritz vectors for component mode synthesis using the singular value decomposition," AIAA Journal, 1996, 34-6, pp 1256-1260

[5] Kerschen G, Golinval JC, Vakakis AF, Bergman LA. The method of proper orthogonal decomposition for dynamical characterization and order reduction of mechanical systems: an overview. Nonlinear dynamics, 2005, 4, pp147-169.

[6] Amsallem, D., Cortial, J., Carlberg, K., and Farhat, C., A Method for Interpolating on Manifolds Structural Dynamics Reduced-order Models, International Journal for Numerical Methods in Engineering, 80, 9, 2009, pp1241-1258.

[7] Balmès, E., "Parametric families of reduced finite element models. Theory and applications." Mechanical Systems and Signal Processing, 1996, 10-4, p. 381-394

[8] Abid F. Chevallier G. Blanchard J.L. Dion J.L. Dauchez N., System identification using Kalman Filters, XXXI IMAC 2013, Garden Grove, California.

[9] Quinn, D. D. Modal analysis of jointed structures, Journal of Sound and Vibration 331.1 2012, pp. 81-93.

[10] Festjens H. Chevallier G. Dion H. Model Order Reduction of assembled Structures for dynamic analysis, submitted to ASME IDETC 2013, Portland.

[11] Kalman R. E. 1960. A new approach to linear filtering and prediction Problems. Transactions of the American Society of Mechanical Engineers - Series D - Journal of Basic Engineering 82, 35-45

[12] Boyce, W.E. and DiPrima, R.C. Elementary differential equations and boundary value problems. John Wiley \& Sons Inc, 1977.

[13] Dormand, J.R. and Prince, P.J. A family of embedded runge-kutta formulae. Journal of Computational and Applied Mathematics, 6(1):19-26, 1980.

[14] Mathews, J.H. and Fink, K.D. Numerical methods using MATLAB. Prentice Hall, 2004. 
ANNEX A

\section{DETAILS OF FIRST ORDER STATE SYSTEM}

The solution of Eq. 22.a on the time interval [tk tk+1] is:

$x^{k+1}=x^{k} e^{F \Delta T}+\int_{t_{k}}^{t_{k+1}} e^{F\left(t_{k+1}-\tau\right)} B u(\tau) d \tau$

Supposing $u(t)$ constant over the sampling interval [tk tk+1], the discrete state space model is written as follows:

$$
\begin{aligned}
& \left.\begin{array}{l}
x^{k+1}=\tilde{F} x^{k}+\tilde{B} u^{k} \\
z^{k+1}=\tilde{C} x^{k+1}
\end{array}\right\} \tilde{F}=e^{F \Delta T} \\
& \tilde{B}=\int_{t_{k}}^{t_{k+1}} e^{F\left(t_{k+1}-\tau\right)} B d \tau=\int_{0}^{T} e^{F \tau} B d \tau ; \tilde{C}=C
\end{aligned}
$$

Where

$$
\begin{aligned}
& \tilde{F}=\left[\begin{array}{ll}
e^{a_{1} \Delta T} & \\
& e^{a_{1} \Delta T}
\end{array}\right] ; \tilde{B}=\left[\begin{array}{l}
\frac{b_{1}}{a_{1}}\left(e^{a_{1} \Delta T}-1\right) \\
\frac{b_{2}}{a_{2}}\left(e^{a_{2} \Delta T}-1\right)
\end{array}\right] ; \\
& \tilde{C}=\left[\begin{array}{ll}
c_{11} & c_{12} \\
c_{21} & c_{22}
\end{array}\right]
\end{aligned}
$$

